\title{
IDENTIFICATION OF SPRING WHEAT GERMPLASM RESISTANT TO POLLUTION OF COPPER AND LEAD FOR THE DEVELOPMENT OF ECOLOGICALLY CLEAN TECHNOLOGY
}

\author{
R. A. Alybayeva ${ }^{1}$, A. S. Aitimova ${ }^{1}$, G. G. Abdrazakova ${ }^{1}$, D. A. Tulebekova ${ }^{1}$ \\ ${ }^{1}$ Al-Farabi Kazakh National University, Department of UNESCO chair for Sustainable development, Almaty, \\ Kazakhstan
}

\begin{abstract}
The creation and usage of technologically resistant breeds of agricultural plants in production is an effective way of solving the problem of soil contamination by heavy metals. At the first stage of this process it's necessary to study the gene pool of cultural and wild-growing plants and to allocate forms which accumulate the minimum quantity of pollutants in a commodity part of a harvest. Objects of an experiment are various spring wheat genotypes from a collection of East Kazakhstan agricultural scientific research institute. During the research growth indicators were identified, the index of tolerance of plants was calculated, as well as the regularity of accumulation and distribution of heavy metals on spring wheat bodies in the conditions of the increased contamination of the environment have been studied. Screening of spring wheat breeds in the laboratory conditions allowed to identify resistant and sensitive genotypes. By indicators of sprouts and roots' growth, the genotype GEK 2082/1 was the most resistant to the influence of lead. The genotype Lutescens 718 - is the least resistant genotype. By indicators of sprouts and roots' growth, the genotype GEK 2077/1 was the most resistant genotype to the influence of copper. The least resistant genotype is GEK 2033/5.
\end{abstract}

\section{Introduction}

Keywords: Heavy Metals, Wheat, Growth Parameters, Resistant Genotypes

One of the most important environmental problems is the contamination of soils by heavy metals (HM). It has an impact on almost all biosphere components.

The main sources of heavy metals contamination of Kazakhstan's topsoil are the enterprises of ferrous and nonferrous metallurgy, mining and processing industry, thermal power stations, transport et al. [1]. That is why contamination of soils by heavy metals has become one of the most important environmental problems in the country, especially in large metropolitan cities and industrial centers. In the industrial regions of the country there are significant foci of anthropogenic pollution of the topsoil.

In addition to such factors as type of soil; concentration; form of the location of $\mathrm{HM}$; soil $\mathrm{pH}$ and its granulometric composition; content of organic substances, cation absorption capacity in soil; presence of technogenic sources of ecosystem pollution, the level of accumulation of heavy metals by plants also depends on their genetic and species features [2].

The study of the features of wheat resistance to heavy metals and identification of germplasm, which has resistance to their impact, is an important step towards the formation and usage of breeds which are resistant to anthropogenic impact in production. For this reason, it is necessary to study the gene pool of cultivated plants and identify the forms that accumulate the minimum amount of pollutants.

The aim of our study is to identify the genetic resistance potential of spring wheat to copper and lead in order to identify metal-resistant forms and donors for selection for metal resistance.

\section{Methods}

The objects of research are: the genotypes of spring wheat GEK 2077/11, GEK 2033/5, Lutescens 718, GEK 2071/8, GEK 2082/1. For the experiment, these plant samples were taken from the collection of the East Kazakhstan agricultural scientific research institute.

Wheat genotypes grew on a nutrient mix that contained $0.1 \mathrm{mM}$ of $\mathrm{CaSO}_{4}$ and $\mathrm{Cu}$ ions at $400 \mathrm{mg} / \mathrm{l}$ concentration (as a $\mathrm{CuSO}_{4}$ salt) or $\mathrm{Pb}$ at $400 \mathrm{mg} / \mathrm{l}$ concentration (as a $\mathrm{PbSO}_{4}$ salt) under model contamination conditions for 14 days. 
Samples were grown in water medium at a t-20 $\mathrm{C}$ during the day and $16^{\circ} \mathrm{C}$ at night, with 10 -hour photoperiod, light intensity - 5 thousand $1 x$, humidity $-60 \%$.

Measurement of growth indicators was carried out according to generally accepted methods. The tolerance index or the Wilkins coefficient was calculated [3].

The method of atomic absorption spectrophotometry was used to determine the concentration of heavy metals [4].

\section{Results}

A study of the genotypic specificity of wheat at resistance to the impact of heavy metals was carried out for copper and lead, the most priority pollutants in the East Kazakhstan region. Since the direct negative effects of heavy metals on plants is manifested in a suppression of the growth and development (attenuation of growth of shoots and roots), indexes of growth processes of wheat genotypes were investigated. This allowed to identify specific features of toxicity of certain metals depending on different genotypic differences of wheat plants.

Our studies of the influence of lead on the growth parameters of wheat germs in laboratory conditions showed that the ions of copper suppress the plant growth (Figure 1).

During the study of genotypes from the collection of the East Kazakhstan agricultural scientific research institute (EKASRI) it was found out that according to the results of study of the above-ground organs' growth with a high concentration of lead in the growth medium, the genotypes can be arranged as follows: GEK 2082/1 > GEK 2071/8 > GEK 2033/5 > GEK 2077/1 > Lutescens 718 (Figure 2).

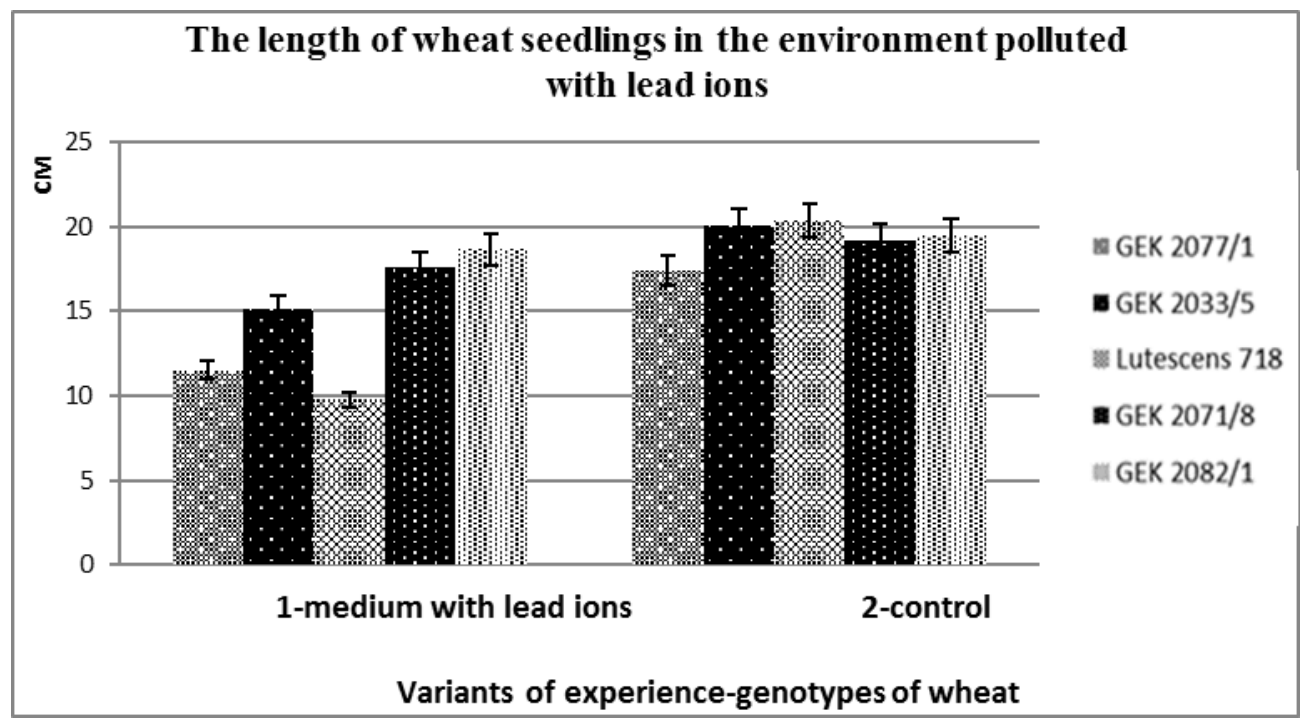

Figure 1: The influence of the presence of ions of lead in the growth medium on the growth of seedlings of different spring wheat genotypes

The most resistant to the adverse effect of lead ions, on indicators of growth of above-ground organs, are genotypes GEK 2082/1 and GEK 2071/8. In genotypes of GEK 2082/1 and GEK 2071/8, the growth of the aerial organs is inhibited to a lesser degree than in the other genotypes when the lead salt is introduced into the growing medium. The suppression of growth seedlings of these genotypes is 4,2 and 8,5 percent, respectively, compared to control (Figure 2). 


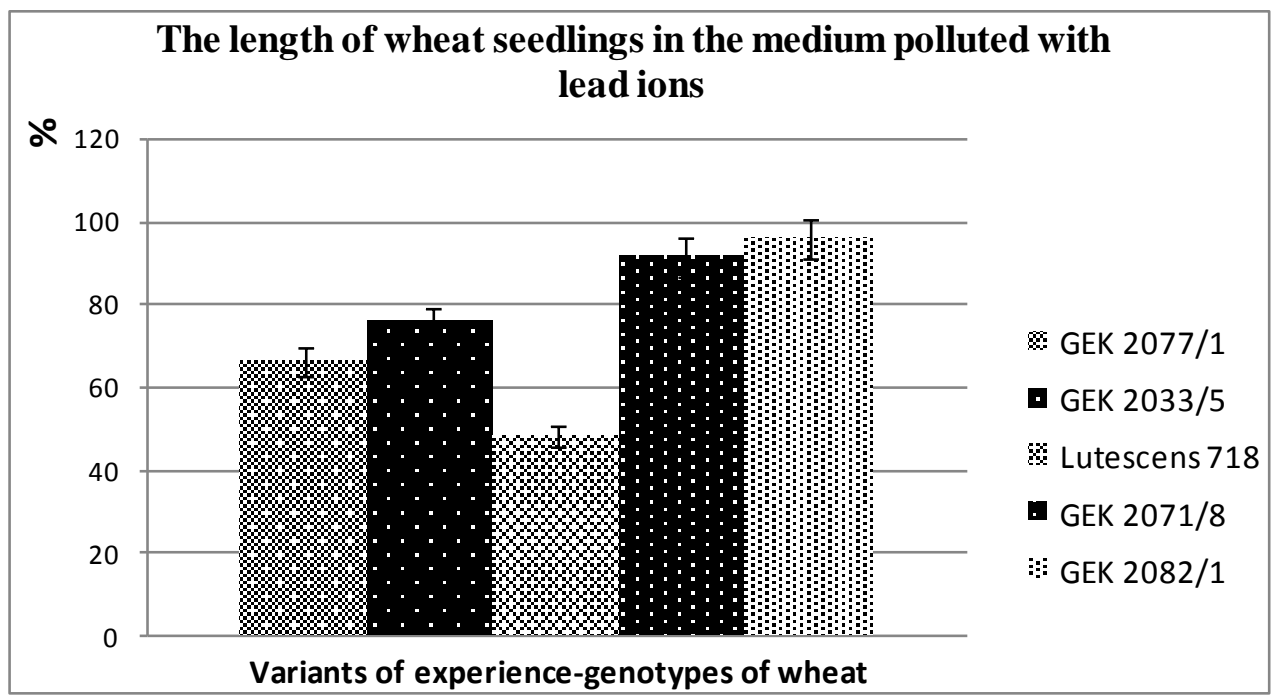

Figure 2: Reduction in the length of seedlings of different wheat genotypes (in percents to control) under conditions of contamination of the medium with lead ions

The average level of resistance to negative influence of ions of lead are in genotypes GEK 2033/5 and GEK 2077/1. The suppression of seedling growth of these genotypes, compared with control - 24.5 and 33.8 percent, respectively in the growth medium polluted by lead salt.

According to the growth of seedlings, the most vulnerable to adverse effect of lead ions is the spring wheat variety Lutescens 718. The growth of seedlings of this spring wheat variety is suppressed in comparison with the control by 52.1 percent (Figure 3).

Thus, according to the growth parameters of the above-ground organs, the genotypes GEK 2082/1 and GEK 2071/8 proved to be the most resistant to unfavorable influence of lead ions, the Lutecens 718 spring wheat variety was the most unstable to the adverse effect of lead ions.

Our studies of the effect of lead on the growth parameters of wheat roots in the laboratory conditions showed that lead ions inhibit the growth of plant roots. Herewith, root growth suppression is more significant than the suppression of seedling growth (Figures 3 and 1).

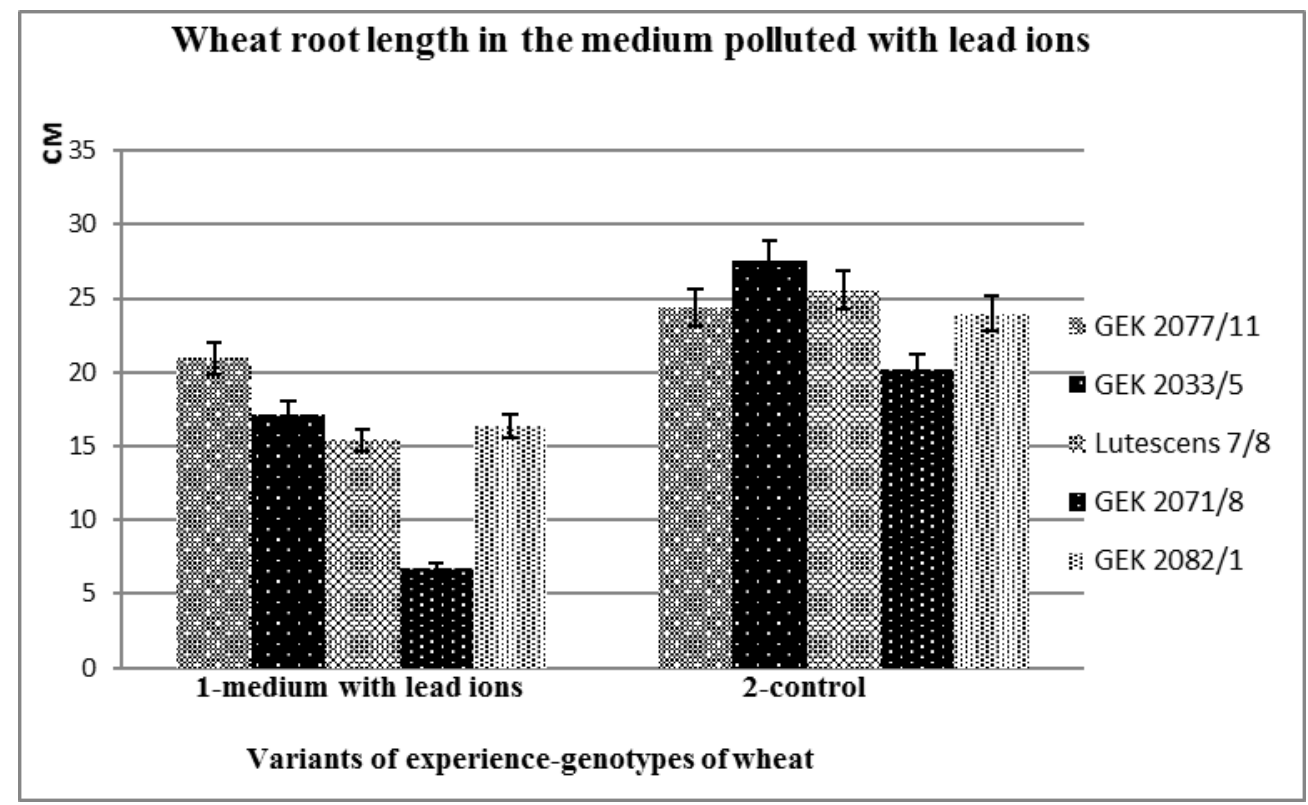

Figure 3: The influence of the presence of lead ions in the medium of growth on root growth of different genotypes of wheat

According to root growth at the time of introduction of the lead salt into the feeding medium, the genotypes can be arranged as follows: GVK 2077/1 > GVK 2082/1 > GVK 2033/1 > Lutescens 718 > GVK 2071/8 (Figure 4). 
The root system of GEK 2077/1 genotype proved to be the most resistant to the adverse effect of lead ions.

This spring wheat genotype's root growth is inhibited to much lesser extent than other genotypes' with a high concentration of lead ions in the medium of growth. Suppression of root growth in comparison with control is 14.2 percent.

It was found that the wheat genotypes GEK 2082/1, GEK 2033/1 and Lutescens 718 have an average level of root resistance to adverse effects of lead. In the meduim contaminated with lead ions, the root growth inhibition in these genotypes is $39.7,37.6$ and 31.6 percent, respectively, compared to the control.

The roots of wheat genotype GEK 2071/8 turned out to be the most unstable to unfavorable impact of lead ions. The root growth inhibition in comparison with control occurs at 66.6 percent (Figure 4).

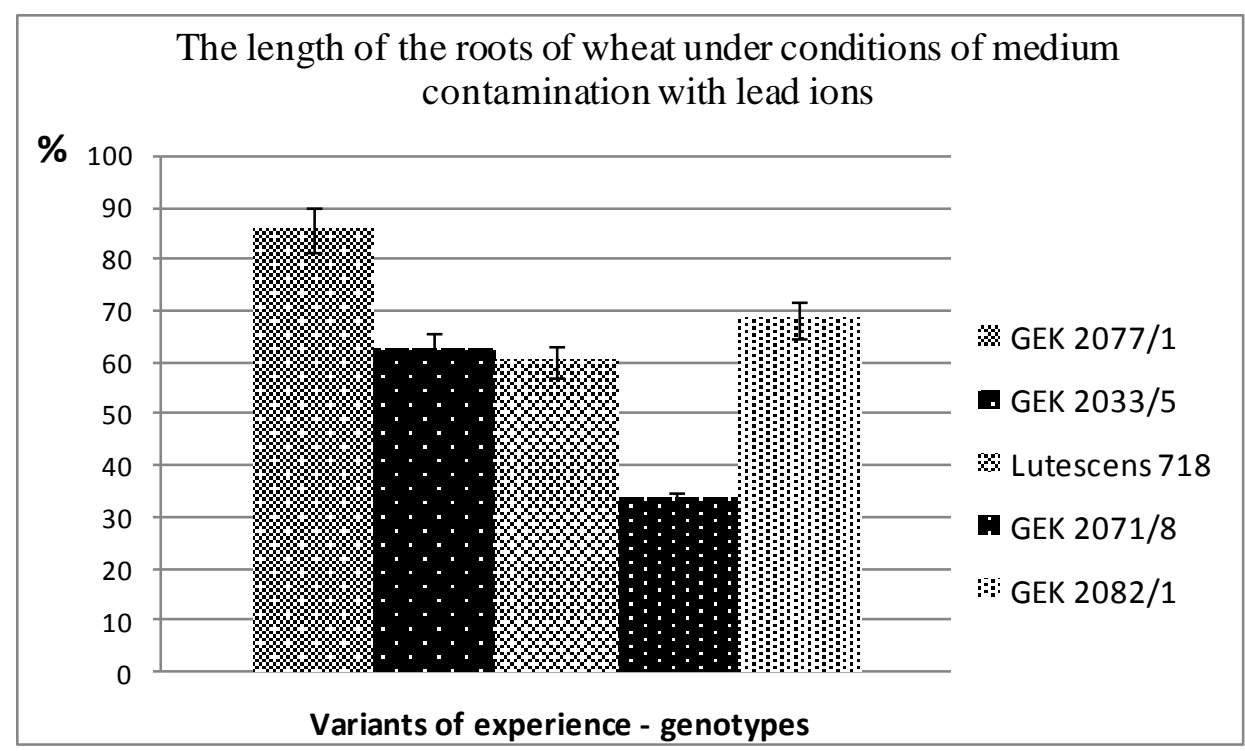

Figure 4: Decreasing of root length of different wheat genotypes (in\% to control) under conditions of contamination of the medium with lead ions.

The Wilkins coefficient was also determined, which shows plant tolerance to heavy metals and is calculated by the formula $I_{t}=I_{m e} / I_{c}$, where $I_{m e}$ is the root increment in the solution with the studied metal, and $I_{c}$ is the root increment in the solution without metal (Table 1).

Table 1 - Wilkins coefficient or tolerance index of roots of wheat seedlings in conditions of environment of growth contaminated by lead ions

\begin{tabular}{lccccc}
\hline Wheat & GEK & GEK & Lutescens & GEK & GEK \\
genotypes & $2077 / 1$ & $2033 / 5$ & 718 & $2082 / 1$ & $2071 / 8$ \\
\hline $\mathrm{I}_{\text {me }}$ & 20,95 & 17,16 & 15,41 & 16,38 & 6,75 \\
\hline $\mathrm{I}_{\mathrm{c}}$ & 24,40 & 27,52 & 25,54 & 23,95 & 20,23 \\
\hline $\mathrm{I}_{\mathrm{t}}$ & 0,86 & 0,62 & 0,60 & 0,68 & 0,33 \\
\hline
\end{tabular}

The highest Wilkins coefficient or tolerance index at a high concentration of lead ions in the growth medium has GEK 2077/1 genotype, the average one - GEK 2082/1 genotypes, GEK 2033/5 and Lutescens 718, the lowest - GEK 2071/8 (Table 1).

According to the results of study of the root growth in contaminated by copper ions medium and the tolerance index, the genotype GEK 2077/1 can be identified as a genotype with the most resistant root system to adverse effect of lead ions.

The average level of root resistance to adverse effects of lead, both on the growth of roots with contamination of the medium by copper ions, and on the tolerance index was revealed in wheat genotypes of GEK 2082/1, GEK 2033/1 and Lutescens 718. 
The most unstable to the adverse effect of lead ions on the basis of the results of the ascertainment of both indicators were the roots of plants of genotype GEK 2071/8.

Thus, according to the results of study of the root growth the GEK 2077/1 genotype proved to be the most resistant to the action of lead ions, the genotype of GEK 2071/8 was the most unstable to unfavorable action of this metal. According to the results of study of the above-ground organs growth, the Lutecens 718 spring wheat sort is most unstable to the adverse effect of lead ions, GEK 2082/1 and GEK 2071/8 genotypes turned out to be the most resistant to lead ions. Our studies of the effect of copper on the growth parameters of wheat seedlings under the conditions of the model experiment have shown that copper ions suppress plant growth (Figure 5).

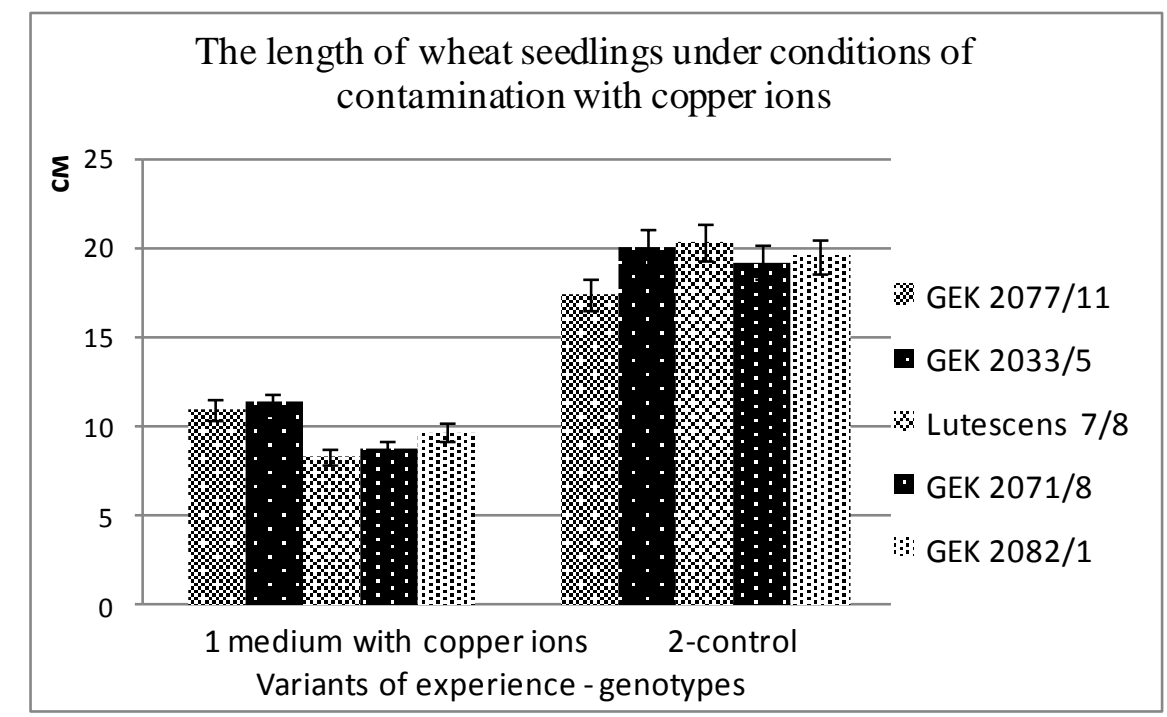

Figure 5: Influence of the presence of lead ions on the growth of seedlings of different wheat varieties in the medium of growth

Wherein suppression of growth of spring wheat seedlings under conditions of contamination of the medium with copper ions is more significant than the suppression of seedling growth under conditions of contamination with lead ions (Figures 5 and 1).

When studying the effect of copper on the growth parameters of seedlings of different genotypes from the EKASRI collection, it was shown that according to the growth of the above-ground organs with high concentration of copper in the growth medium, the genotypes can be arranged as follows: GEK 2077/1 and GEK 2033/5> GEK 2082/1> GEK 2071/8> Lutescens 718 (Figure 6). 


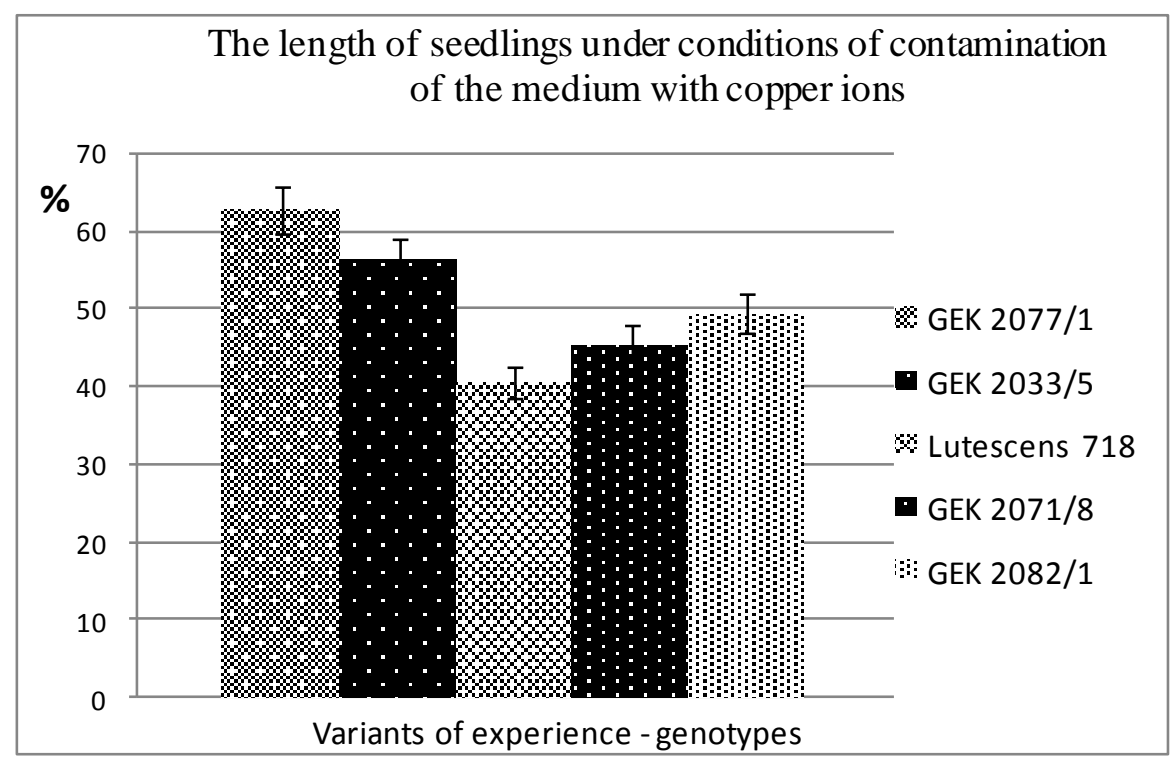

Figure 6: The reduction of the length of seedlings of different wheat genotypes (in $\%$ to control) under conditions of contamination of the medium with copper ions

The genotypes of spring wheat GEK 2077/1 and GEK 2033/5 proved to be the most resistant to unfavorable influence of copper ions, according to the results of study of growth indices of above-ground organs. The suppression of seedlings growth during cultivation in an medium contaminated with copper ions compared to the control occurs at 37.2 and 43.2 percent, respectively.

The growth of the above-ground organs of the genotypes of spring wheat GEK 2082/1 and GEK 2071/8 is inhibited in an average degree in comparison with other genotypes at the time of addition of copper salt into the growth medium.

Suppression of growth of seedlings of these genotypes in comparison with the control occurs at 54.4 and 50.4 percent, respectively.

The most unstable to unfavorable action of copper were the above-ground organs of the spring wheat variety Lutescens 718. Suppression of growth of seedlings in comparison with the control occurs at 59.3 percent (Figure 6). Our studies of the copper impact on the growth parameters of the roots of wheat in laboratory conditions showed that copper ions inhibit root growth. Furthermore, the root growth suppression is more significant than suppression of seedling growth (Figures 7 and 5). 


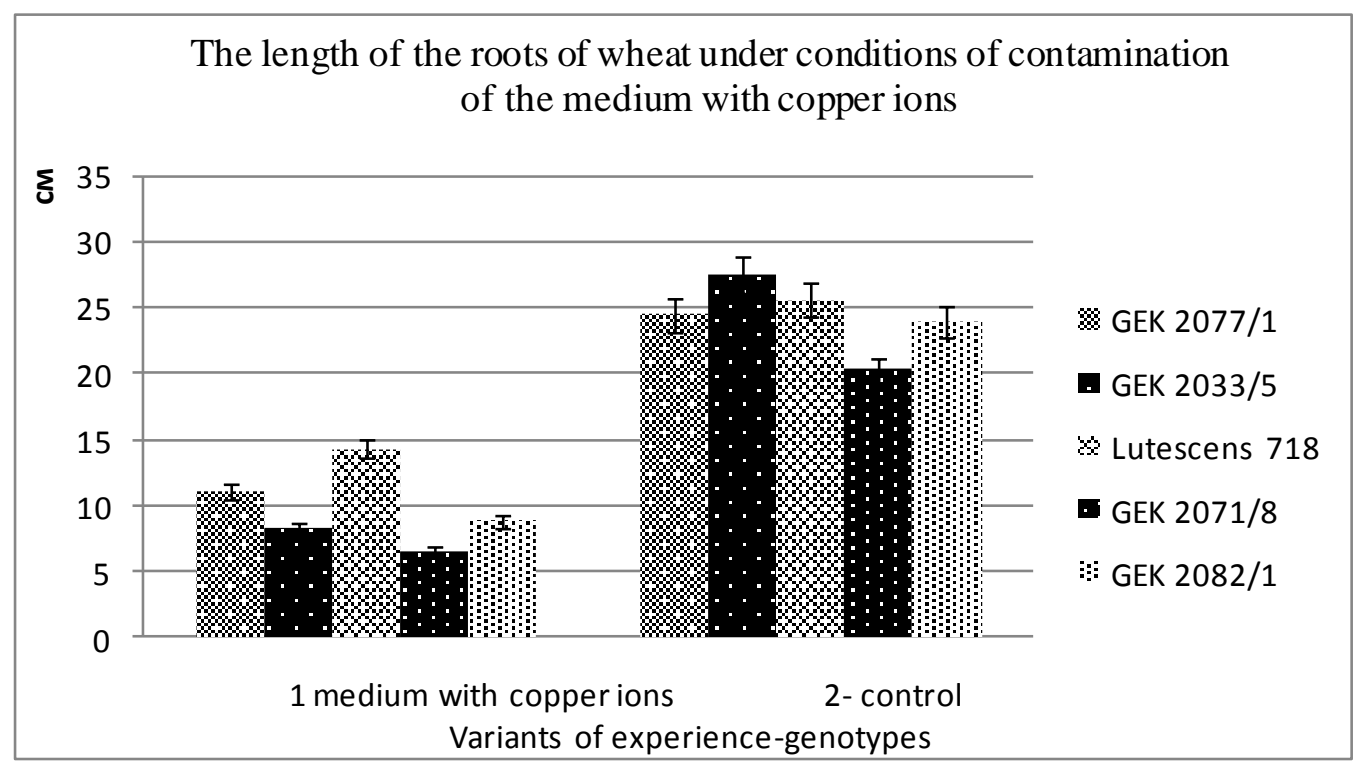

Figure 7: The influence of the presence of copper ions in the growth medium on root growth of different wheat genotype

Studies of the effect of copper on plant growth parameters of genotypes from the EKASRI collection have shown that genotypes can be arranged according to the study of root growth at a high concentration of copper ions as follows: Lutescens 718> GEK 2077/1> GEK 2082/1> GEK 2071/1> GEK 2033 / 5 (Figure 8).

The root system proved to be the most resistant to the unfavorable effect of copper in the genotypes of spring wheat Lutescens 718 and GEK 2077/1.. When copper is entered into the growing medium, root growth is less inhibited in these genotypes than in other genotypes. The inhibition of root growth occurs 44.2 and 54.9 percent compared to control, respectively (Figure 8).

The genotype of spring wheat GEK 2082/1 has the average level of resistance of the roots in the conditions of contamination of the growing medium by copper ions. The suppression of root growth occurs at 63.4 percent compared to control, respectively.

The roots of the spring wheat genotypes GEK 2071/8 and GEK 2033/5 were the most unstable to the adverse effects of copper. The suppression of root growth occurs 67.9 and 70.1 percent respectively compared to the control in medium contaminated by copper ions (Figure 8). 


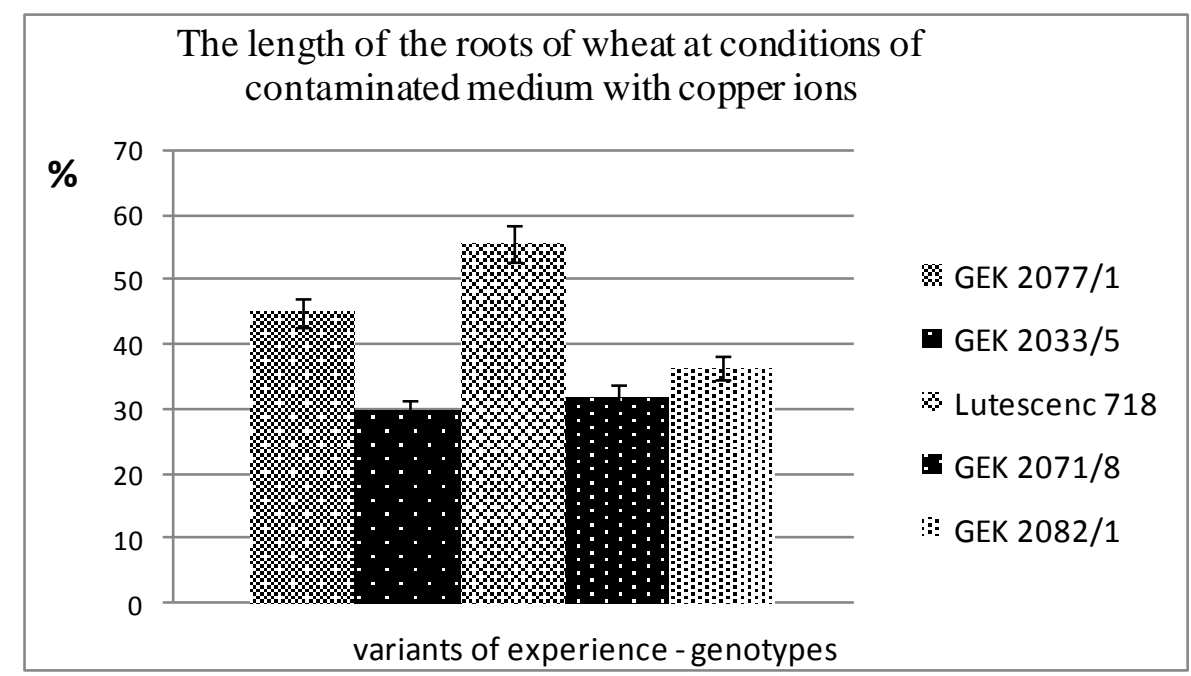

Figure 8: Reduction of root length of different spring wheat genotypes (in percents to control) in conditions of medium contaminated with copper ions

The Wilkins coefficient was also determined in the case of studying the influence of copper on wheat growth parameters, as well as in the case of the study of the responses of wheat plants of different genotypes to the adverse effect of lead ions in the growing medium (table 2).

Table 2 - The Wilkins coefficient or tolerance index of wheat roots in terms of contamination by copper ions

\begin{tabular}{lllllllc}
\hline $\begin{array}{l}\text { Wheat } \\
\text { genotypes }\end{array}$ & GEK & GEK & GEK & Lutescens & GEK & GEK & GEK \\
\hline $\mathrm{I}_{\text {me }}$, & $2077 / 1$ & $2077 / 1$ & $2033 / 5$ & 718 & $2071 / 8$ & $2082 / 1$ & $2071 / 8$ \\
\hline $\mathrm{I}_{\mathrm{c}}$ & 11,01 & 11,01 & 8,23 & 14,25 & 6,50 & 8,77 & 6,75 \\
\hline $\mathrm{I}_{\mathrm{t}}$ & 24,40 & 24,40 & 27,52 & 25,54 & 20,23 & 23,95 & 20,23 \\
\hline
\end{tabular}

At a high copper concentration Wilkins coefficient or tolerance index is the highest at Lutescens 718 , average is in GEK 2077/1, GEK 2082/1 and least is in GEK 2033/5 and GEK 2071/8 (Table 2).

According to the study of root growth and the tolerance index, genotypes Lutescens 718 and GEK 2077/1 have the most resistant root system to the adverse effects of copper ions.

The average level of resistance of the roots to the unfavorable effect of copper, both on the growth of roots with contamination of the medium by copper ions, and the tolerance index was revealed in wheat genotypes of GEK 2082/1.

According to the results of the determination of both indicators, the roots of the genotypes of GEK 2033/5 and GEK 2071/8 turned out to be the most unstable to the adverse effect of copper ions. 
Thus, the genotypes of spring wheat Lutescens 718 and GEK 2077/1 proved to be the most resistant according to root growth, the genotypes of GEK 2033/5 and GEK 2071/8 proved to be the most unstable to the adverse effect of copper. According to the growth of the above-ground organs, the genotypes of spring wheat GEK 2077/1 and GEK 2033/5 proved to be the most resistant to the action of copper, Lutescens 718 is the least resistant.

The plants of genotype GEK 2077/1 show root stability and stability of above-ground organs to unfavorable action of copper ions. The studying of the patterns of accumulation and distribution of copper and lead in the organs of spring wheat depending on the genotypic differences in the samples at the conditions of the model experiment was carried out.

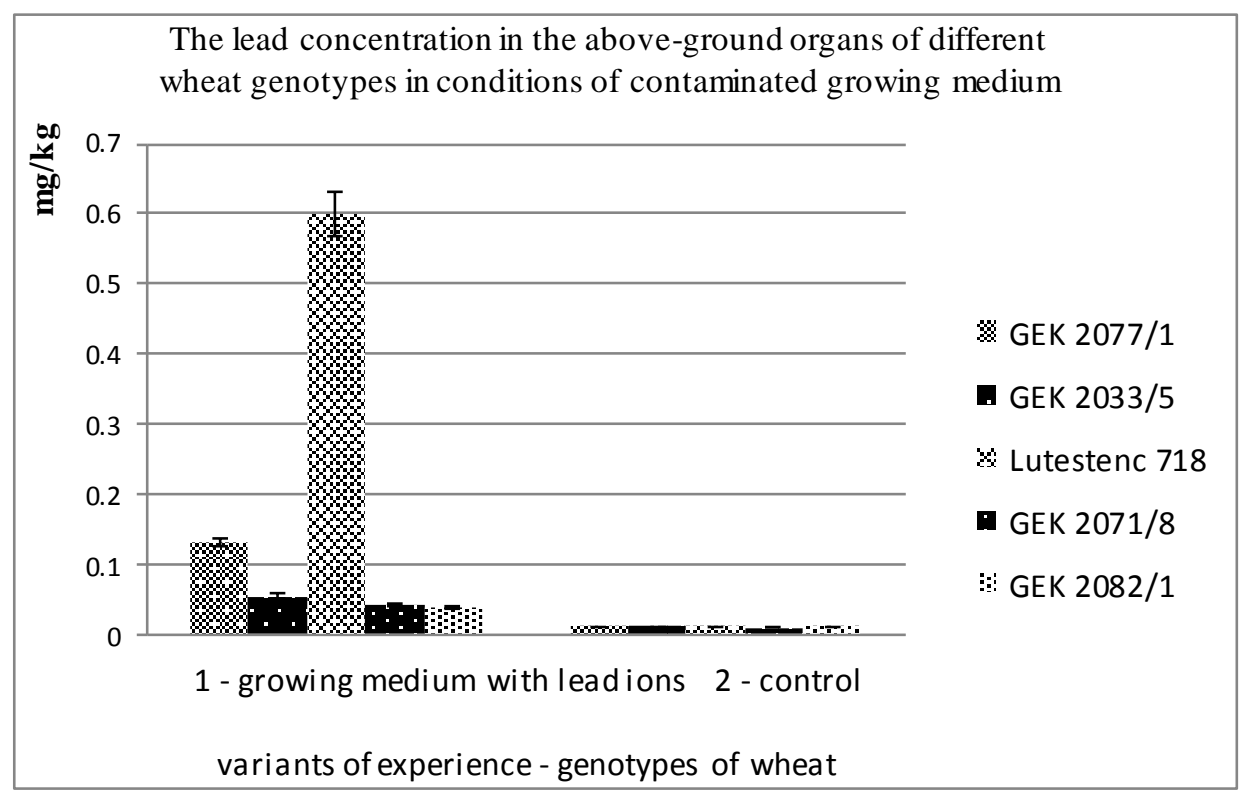

Figure 9: The lead content in the above-ground organs of different wheat genotypes in conditions of contaminated growing medium

The results of the determination of the lead content (at a dose of lead in the medium of $400 \mathrm{mg} / \mathrm{l}$ ) showed that the genotypes GEK 2082/1 and GEK 2071/8 accumulated the smallest amount in above-ground organs. The seedlings of the spring wheat breed Lutescens 718 accumulate the largest amount of lead in the above-ground organs.

The remaining genotypes occupy an intermediate position between them - GEK 2077/1 and GEK 2033/5 (Figure 9). Thus, the GEK 2082/1 and GEK 2071/8 showed the greatest resistance of lead intake in the above-ground organs, the largest amount of lead in the above-ground organs is in genotype of spring wheat Lutescens 718 (Figure 9).

A study of the lead content, at a dose of lead in a medium of $400 \mathrm{mg} / \mathrm{l}$, of different spring wheat genotypes showed that GEK 2082/1 accumulated the least amount in the roots. In these experimental conditions, the highest amount of lead accumulated in the roots of spring wheat GEK 2071/8. The remaining varieties occupy an intermediate position between them - a breed of spring wheat Lutescens 718 and genotypes of spring wheat GEK 2033/5 and GEK 2077/1.

Thus, the least accumulation of lead is observed in the roots of spring wheat genotype GVK 2082/1, the plants of spring wheat GEK 2071/8 accumulate the largest amount of lead in the roots (Figure 10).

According to the study results, genotype GEK 2082/1 can be distinguished as a genotype with the greatest root resistance to lead. The sprouts of spring wheat genotypes GEK 2082/1 and GEK 2071/8 have shown as genotypes resistant to translocation of lead into the above-ground organs. 


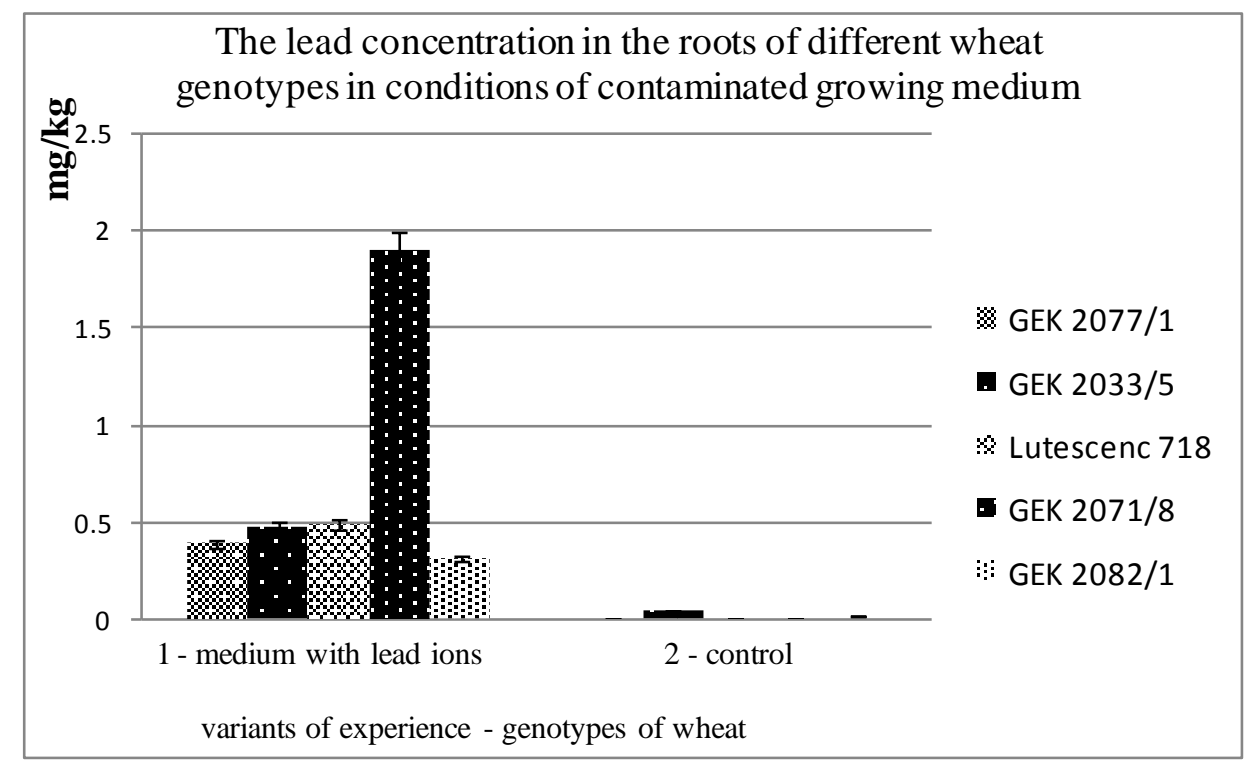

Figure 10: The lead content in the roots of different wheat genotypes in conditions of contaminated growing medium

The study of lead accumulation in roots and above-ground organs, of growth parameters of wheat germs of different genotypes made it possible to identify the most sensitive and resistant genotypes. The GEK 2082/1 was most resistant genotype to lead and the most sensitive was spring wheat variety the Lutecens 718 .

The study of the copper content in the above-ground organs of different genotypes of spring wheat showed that the lowest accumulation of copper (its content of $400 \mathrm{mg} / \mathrm{l}$ in the medium) is observed in sprouts of genotype GEK $2077 / 1$.

Genotypes of GEK 2071/8, GEK 2082/1 and GEK 2033/1 are characterized by an average accumulation of copper in the above-ground organs at these experimental conditions.

In the above-ground organs, the greatest content of copper is observed for breed of the Lutescens 718 (Figure 11). Thus, the greatest resistance to the entry of copper into the aerial organs was shown by the genotype of GEK 2077/1, the greatest accumulation of copper is observed in the seedlings of the breed Lutescens 718 and GEK 2071/8 (Figure $11)$.

The study of copper accumulation in roots of plant, (its content in the medium of $400 \mathrm{mg} / \mathrm{l}$ ) showed that the least amount contained in seedling roots of spring wheat breed Lutescens 718 .

Genotypes of GEK 2071/8, GEK 2077/1 and GEK 2082/1 are characterized by an average accumulation of copper in the roots of plants at these experimental conditions. The highest content of copper is observed in the roots of plants of genotype GEK 2033/5 (Figure 12). 


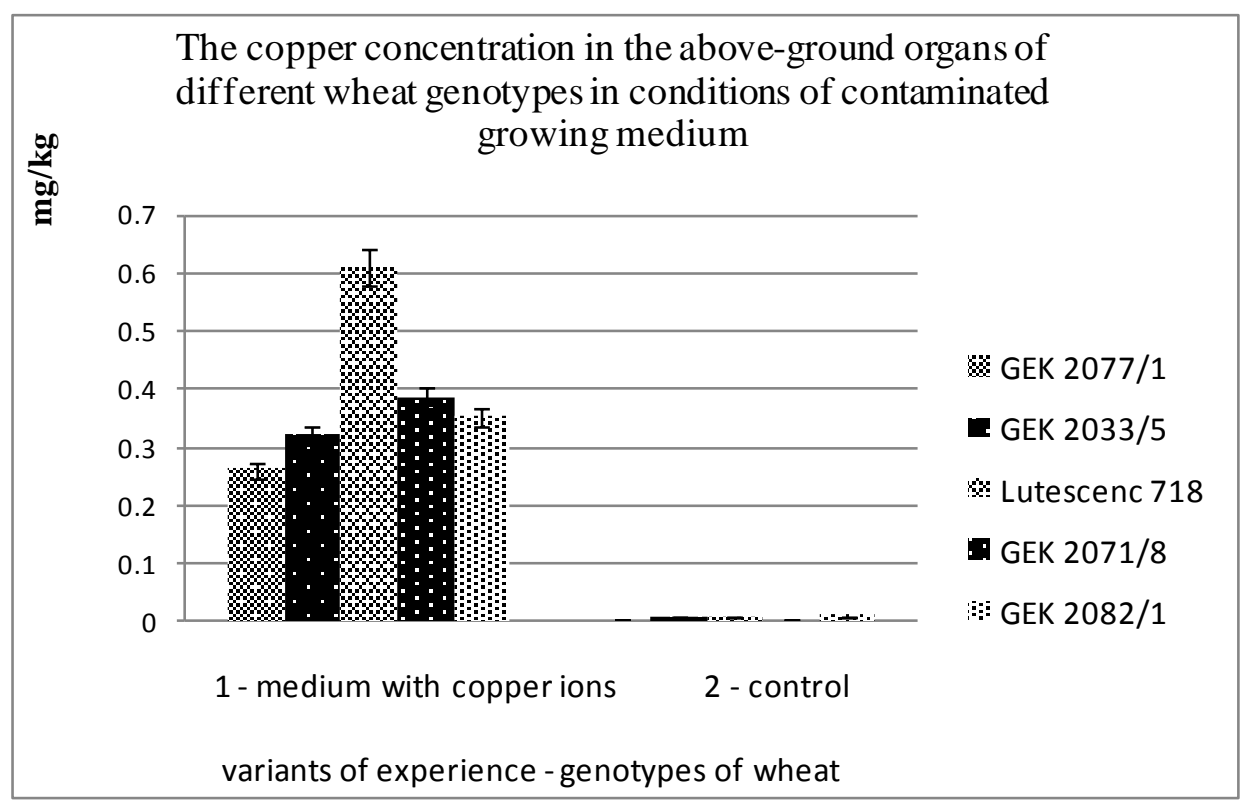

Figure 11: The copper content in the above-ground organs of different wheat genotypes in conditions of contaminated growing medium

Thus, the smallest accumulation of copper is observed in the roots of the spring wheat breed Lutescens 718 , the largest - in the roots of the genotype GVK 2033/5 (Figure 12).

The study of copper accumulation in the roots, above-ground organs and growth parameters allowed to reveal the most sensitive and stable genotypes. The most resistant to the action of copper was the genotype of spring wheat GVK 2077/1, the most unstable to the adverse effect of this metal was the breed of spring wheat Lutescens 718 .

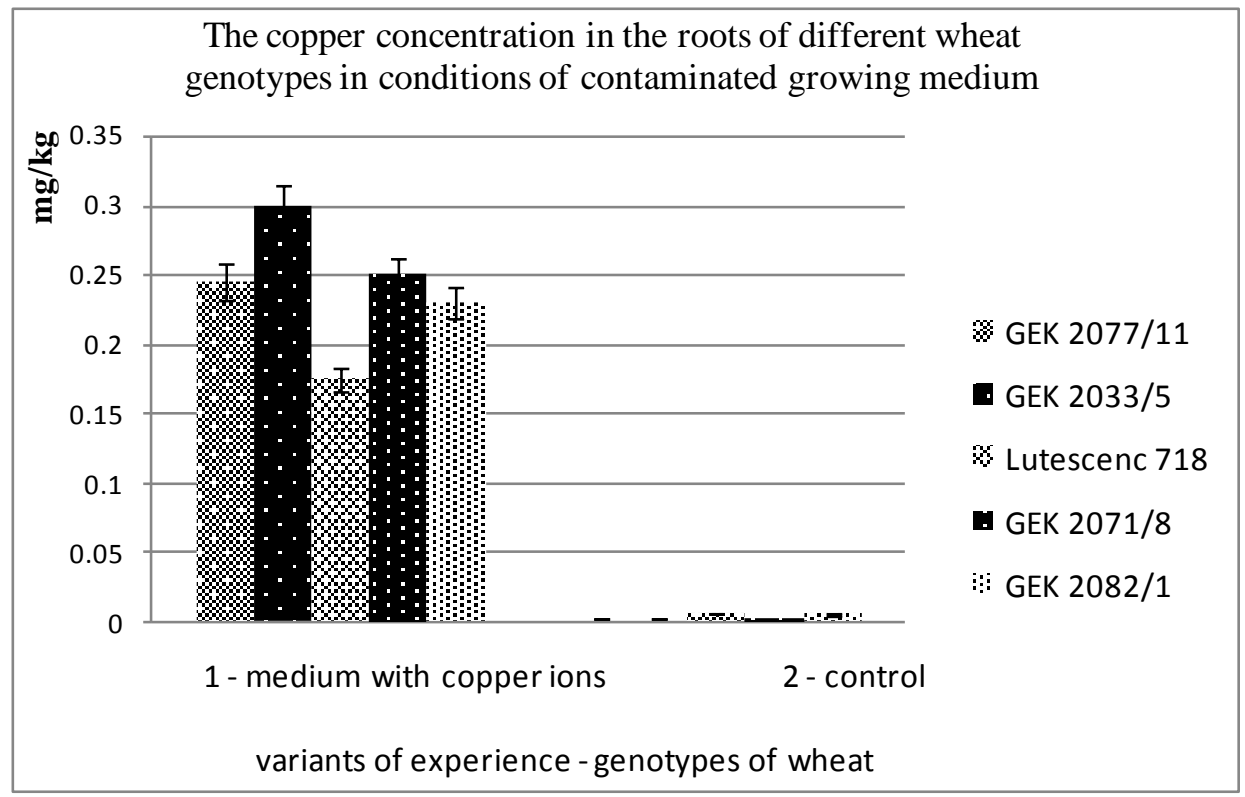

Figure 12: The copper content in the roots of different wheat genotypes in conditions of contaminated growing medium 


\section{Conclusion}

According to the research results, studied genotypes showed a genotypic specificity in relation to the adverse effects of lead and zinc. The above-ground organs and the root system have differential features of accumulation of heavy metals, which in the future may effect on their growth and development parameters. In the roots of the genotype of spring wheat GEK 2082/1 observed the smallest accumulation of lead, plants of the genotype Lutescens 718 accumulate the largest amount of lead in the roots. The genotypes of GEK 2082/1 and GEK 2071/8 showed the greatest resistance to the intake of lead in the above-ground organs, the genotype of spring wheat Lutecens 718 was the least resistant.

The lowest accumulation of copper is observed in the roots of the spring wheat variety Lutescens 718 , the highest in the roots of the genotype GEK 2033/5. Genotype GEK 2077/1 showed the greatest resistance to copper entering the above-ground organs, the greatest accumulation of copper was in sprouts of GVK 2071/8 and Lutescens 718

\section{References}

Chertko N.K. (2002) Geochemical ecology, Tutorial, BGU 79

Panin M.S. (1999) Accumulation of heavy metals by the plants of Semipalatinsk Irtysh region. Semipalatinsk,

Koval S.F. (1974) Investigation of the cell membranes properties and the resistance of plants by elution of electrolytes // // News of the Sib. Branch of the Academy of Sciences of the USSR. Series of Biol.Sciences, 15 (3) 161-167

Perelman A.I. (1989) Geochemistry, Moscow: Higher School 527 